\title{
Psychophysiological mechanisms for learning tasks solving of different complexity
}

\author{
Anna Fomina ${ }^{1, *}$, Oksana Kundupyan ${ }^{2}$, Artem Starostin $^{2}$ and Mikhail Bibov ${ }^{3}$ \\ ${ }^{1}$ Don State Technical University, Russia \\ ${ }^{2}$ Southern Federal University, Russia \\ ${ }^{3}$ Rostov State Medical University, Russia
}

\begin{abstract}
The dynamics of cognitive functions of students of faculties of natural sciences has been analyzed during verbal task solving. EEG and time required for solving of model learning tasks have been registered. Solving of learning tasks was divided into three stages. The first stage was connected with the task reading and preparation for the answer, and was followed by activation of the long-term memory. At the second and third stages, which were connected with formulation of the answer and text writing, the key role belonged to the working memory.
\end{abstract}

\section{Introduction}

Study of neuronal principles of academic activity is presently considered to be one of important problems of modern cognitive science. Introduction of remote learning in higher school requires development of special tools for impartial assessment of student's knowledge and skills. This key angle of this problem is the formation of readiness to solve problem tasks that leads to the development of competence to apply knowledge and skills in unconventional situations. In this situation students interact with interactive educational medium with the feedback from the tutor.

In natural sciences, the parameter of successful learning is the ability of a student to solve model professional tasks. The successful independent work enables a student to adapt effectively to real professional activity. Therefore, differential knowledge control is absolutely crucial.

It is necessary to assess the impact of the main cognitive processes into the formation of knowledge and skills, taking into account the special emphasis on individual learning. For students, who study natural sciences, the retrieve of formerly studied information is associated with activation of prefrontal cortex, whereas obtainment of new theoretical knowledge is connected with activation of temporal cortex [1]. The effective learning also correlates with growth of activity of central cortex zones, which are associated with attention and memory, while ineffective learning is connected with activation of somatosensory zones [2]. This is believed to be connected with more effective formation, strengthening and consolidation synaptic links in the zones of interest [2]. This situation does not occur with

\footnotetext{
*Corresponding author: a_bogun@mail.ru
} 
students of humanity faculties, suggesting the role of educational content in these processes $[1,3]$. This may also be considered as a mechanism of brain adaptation to the studying [2].

According to the modern requirements for the test tasks, no intervention of any kind that may decrease the efficacy of studying is allowed. Hence, the goal of this work was to study the dynamics of cognitive functions of students during model learning process.

\section{Materials and methods}

The study involved 20 third-year students of the D. I. Ivanovsky Academy of biology and Biotechnology, Southern Federal University, of 20 - 22 years of age. The experiments were carried out in a dark soundproof room, in which the individuals tested seated in front of the computer. All subjects were informed about the order of the testing procedures and signed written agreement for the tests to be performed in accordance with ethical standards. The methodology consisted of modeling of real educational process under the conditions of psychophysiological examination. Tasks of different content and complexity, which were similar to those from the courses of "Neurobiology" and "Particular Physiology of Central Nervous System" were developed:

1. The situational task is the simple standard activity, which consisted of writing of an answer for a certain question of either verbal or visual type. Solving of a verbal task required detailed explanation of a physiological phenomenon. The number of terms required was determined by the tutor in advance. It was allowed to substitute the term by a suitable synonym. Three tasks of each type were used.

Visual tasks consisted of images of different parts of central nervous system (CNS) and required to write a correct name for each part. Strictly determined anatomic terminology was used, and substitution for synonyms was not allowed.

2. Competence task implied creative activity that consisted of solving of a problem situation or physiological task either verbal or visual. Verbal tasks included text information only, whereas visual tasks consisted of combination of text with graphics, schemes and images. The basic set of terms was also predetermined in advance. Students were allowed to choose the terms by themselves and/or substitute them with correct synonyms. Three tasks of each type were used.

Answers for all tasks were typed in a standard type MS Word file. Students were provided with the following instruction: press the mouse-manipulator button after reading the task, before starting to type the answer and after finishing the task (Fig. 1). This approach allowed us to identify and analyze the following elements of activity:

1. Reading of the task: the interval from the moment of slide representation to the first press of the mouse-manipulator button. During this interval student read the text and/or analyzed images.

2. Preparation to the text typing: the interval between the first press of the button after the analysis of a slide and the second press before starting to type the answer. During this period student considered the answer.

3. Text typing: the interval between the second and third press of the button. During this period student typed the answer. 


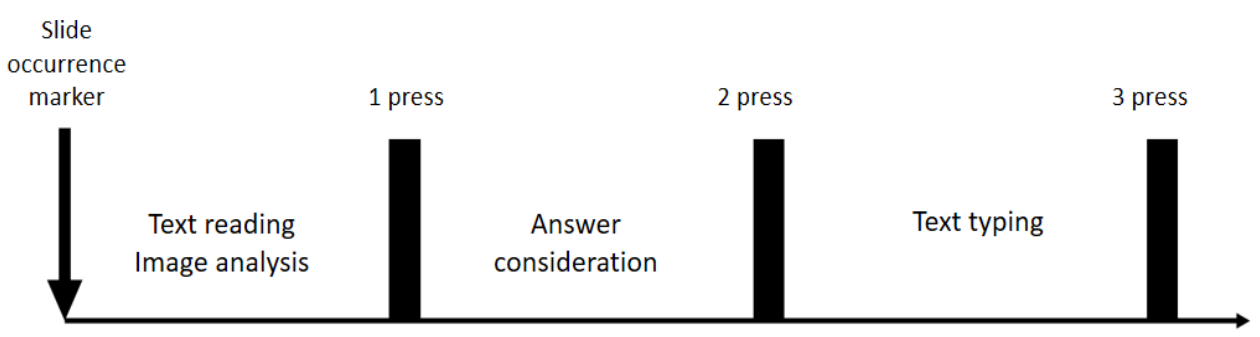

Fig. 1. Stages of a model task solving.

The correctness of studying was assessed by the tutor using the expert mark system.

Stimuli were represented using the "Audiovisual slider" program (Medicom-MTD, Russia) at the grey background in the center of the computer screen at $1 \mathrm{~m}$ distance at the eye level. Electrophysiological parameters were registered with the multichannel computer-based encephalograph "Encephalan-131-03" (Medicom-MTD, Russia). Reference electrodes were placed upon earlaps; the indifferent electrode was located on the forehead. Digitalized EEG, as well as the stimuli and button press markers, were exported into the MATLAB for further processing. Time required to solve individual tasks of each type, duration of each stage, the number of terms used and the values of spectral power of four EEG diapasons were analyzed: delta $(1-3.5 \mathrm{~Hz})$, theta $(4-8 \mathrm{~Hz})$ alpha $(8-13 \mathrm{~Hz})$ and beta $(13-30 \mathrm{~Hz})$.

Spectral power was analyzed by the artifact-free EEG-segments. Confidence of differences was assessed by ANOVA at the significance level 0.05 . Holm and Tukey methods were used to correct multiple comparisons and the Greenhouse-Geisser correction was used to correct the number of freedom degrees. The statistical analysis of the EEG spectral power (SP) was performed for the zones selected: frontal, central, temporal, parietal and occipital. The dispersion analysis was carried out taking into account the following factors: CONTENT (visual or verbal task), COMPLEXITY (situational or competence task) and LOCATION (five zones selected). The factor of the CONDITION, which included the comparative analysis of the text with the background, was considered individually. Individual differences were considered as random factors.

\section{Results}

The correctness of the answer was considered to be the main assessment parameter. Besides the expert mark, the mean number of terms (in \%) predetermined by the tutor was used to assess the answer, because it was required to provide the answer in written form. This parameter indicated the level of theoretical knowledge (situational task) and capacity to apply it to an unconventional situation (competence task). It also allowed us to estimate the number of required special terms used, as well as the number of general scientific terms, which were used to formulate the answer.

The mean number of terms used (in \%) to solve the tasks of all types is shown on the Fig. 2. The statistical analysis revealed significant influence of the factor of the CONTENT on this parameter $(1: 1, \mathrm{~F}=10,398, \mathrm{p}=0,001606)$, whereas the factor of the COMPLEXITY provided no effect. In case of verbal tasks the number of terms used did not depend on the complexity level and was $39,7 \%$ and $37,6 \%$ respectively. Solving of situational tasks required $60,4 \%$ terms, while solving of competence tasks required $48,8 \%$ of terms. However, one should bear in mind that solving of a situational visual task was characterized by high variability, and the percentage of terms used varied from $0 \%$ (if no answer was provided) to $100 \%$ (if the answer was solely correct). 

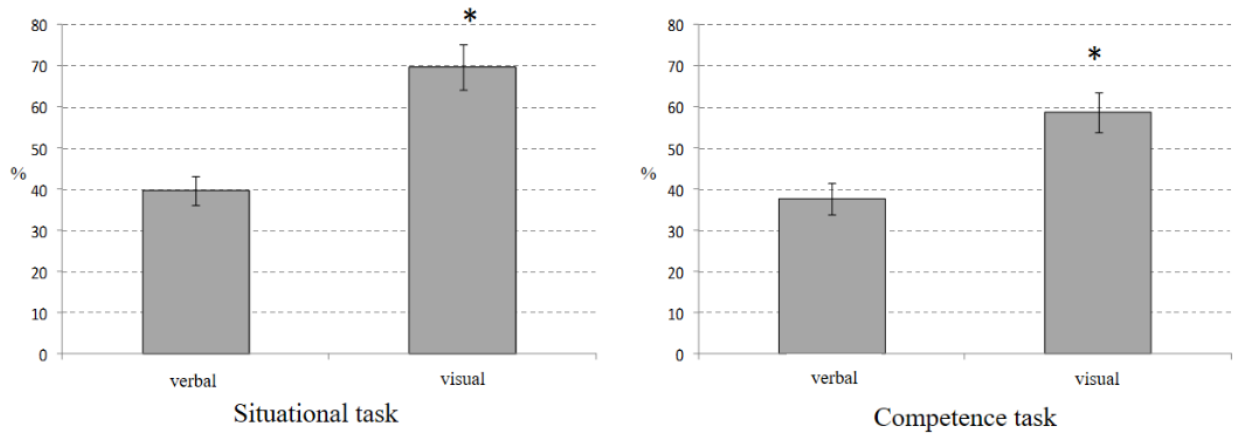

Fig. 2. The mean number of terms used to solve the situational and competence tasks by a group of students. Note: the abscissa shows the task type; ordinate shown the percentage of terms used, $\%$.

The analysis of time required to solve individual tasks of different type revealed significant influence of the factor of the COMPLEXITY $(1: 1, F=7,3712, p=0,007558)$ due to shorter time period used to solve verbal situational tasks as compared with visual ones. No difference in the time used to solve competence tasks of both types was observed.

Therefore, the duration of stages of a task solving is believed to be more informative. The number of these stages varied from 1 to 2 under the conditions of model learning activity. In fact, the activity might have been divided into two stages only: reading of a task and writing of an answer. This is connected with possibility of semantic overlapping of their content due to the beginning of consideration of the answer already during reading of the task. Apparently, if the situation requires switching between the programs used to solve the task, for example if it is necessary to write a text, analyze literature and perform the statistical analysis, the number of these switches may be used as an analogue of the number of operations. This situation remained during the analysis of the first stage of a task solving, which is connected with perception of the task (Fig. 3). Significant influence of the COMPLEXITY factor $(1: 1, F=6,5993, p=0,011367)$, as well as the combined effect of the factors of COMPLEXITY * CONTENT $(1: 1, \mathrm{~F}=6,2093, \mathrm{p}=0,014007)$, were observed. Significantly shorter period of this fragment was observed for the situational verbal task in comparison with others. The duration of this stage during the visual task solving was longer. No significant differences were observed for the competence tasks of both types.

The analysis of the stage 2 revealed confident influence of the COMPLEXITY factor $(1: 1, F=4,7678, p=0,030849)$ owing to increase in duration of this stage in case of verbal competent task solving in comparison with situational ones. For the visual tasks no such dynamics was found.
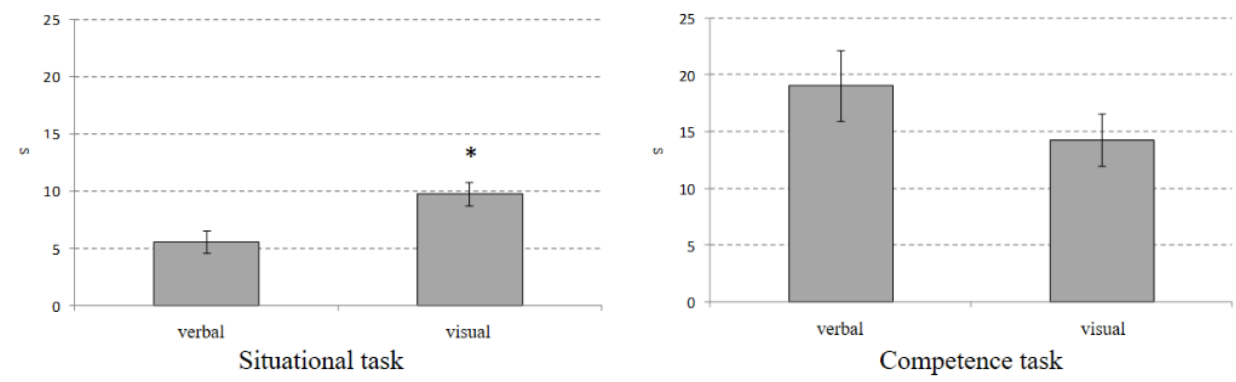

Fig. 3. Mean duration of the first stage of a task solving depending on the task type and complexity. Note: the abscissa shows the task type; ordinate shown the duration of the stages.

The analysis of general dynamics of three EEG frequency diapasons without separation of the stages (Fig. 4) revealed that delta-frequencies predominated quantitatively in the 
spectrum during solving of tasks of any type. Delta-diapason synchronization, as well as significant increase in the quantitative parameters, was observed in comparison with the state of quiet wakefulness. Solving of verbal tasks as associated with significant linear increase in the spectral power alongside with increase in complexity of the tasks. Solving of a situational task led to obvious concentration of oscillations in the frontal-central zone with the shift to the left, whereas solving of the competence tasks was associated with irradiation of oscillations from frontal zones to the posterior ones, making the maximal expression focus obscure.

Quantitative differences were observed for the theta-diapason. Switching from the state of quiet wakefulness with open eyes was followed by significant synchronization of oscillations regardless of task content and complexity. The increase in the task complexity was associated with linear increase in the spectral power the highest values being observed in case of the competence task solving.

Alpha-diapason during the task solving was characterized by significant desynchronization in comparison with the background conditions. The powerful parietooccipital focus, which was present in the background, was preserved for all types of tasks. The differences were quantitative and expressed in the level of its desynchronization. No confident differences were observed for the beta- 1 and beta- 2 diapasons, and therefore, these data are not shown.

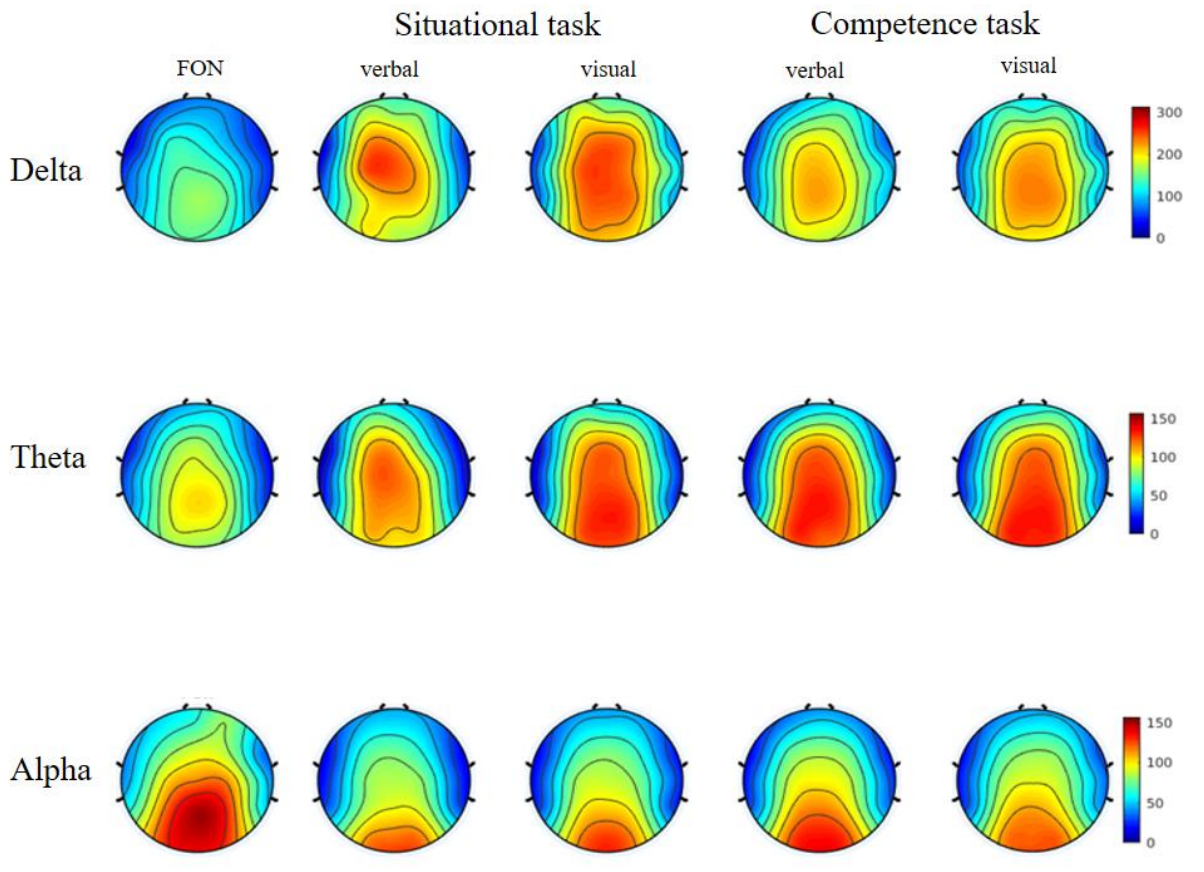

Fig. 4. The topographic maps of the distribution of three spectral EEG diapasons in the state of quiet wakefulness with open eyes and during the task solving of all types. Note: functional probes are shown on the top; the corresponding rhythmic EEG diapason is shown on the left; The dark color indicates high level of spectral power in the leads.

Further analysis of the dynamics of spectral characteristics of the EEG diapasons was performed for stages 1 and 2 of the task solving in comparison with quiet wakefulness with open eyes. Multiple dispersion analysis of the stage 1 revealed significant influence of the CONDITION (4, F=120,79298, $\mathrm{p}<0,001)$ and LOCATION $(20, \mathrm{~F}=12,4867653, \mathrm{p}<0,001)$ factors. 
The single-factor analysis of variance of the dynamics of spectral characteristics of the EEG diapasons at the stage 1 showed significant decrease in the EEG spectral power in comparison with the background level. The dynamics of the delta-diapason at the stages 1 and 2 is shown on the Fig. 5. The single-factor analysis of variance at the stage 1 showed considerable effect of the CONDITION (4, F=103,67, $\mathrm{p}<0,001)$ and LOCATION (20, $\mathrm{F}=14,02, \mathrm{p}<0,001)$ factors.

The stage 1 of the verbal situational task solving was characterized by significantly higher level of the delta-diapason of the EEG spectral power in comparison with visual situational tasks ( $p=0,000017$ ). Opposite dynamics was observed for the competence tasks, for which the values of the delta-diapason of the EEG spectral power were confidently higher in case of visual tasks $(p=0,000164)$. Analysis of the influence of the task complexity showed that solving of the competence tasks was characterized by lower EEG spectral power in comparison with situational ones regardless of the task content $(p=0,00001)$.

Different situation was observed for the stage 2 . A significant influence was also observed for the factors of CONDITION $(4, \mathrm{~F}=114,47, \mathrm{p}=0,00)$ and LOCATION (20, F=16,73, $\mathrm{p}<0,001)$. The single-factor analysis of variance, which was carried out in order to compare tasks of different type, showed that verbal situational tasks were characterized by lower EEG spectral power in the delta-diapason in comparison with the stage $1(1,1, \mathrm{~F}=42,09, \mathrm{p}<0,001)$. No difference was observed for the stages 1 and 2 of visual situational task solving. Differences observed for the competence tasks were associated with visual tasks, which were also characterized by low EEG spectral power at the stage $2(1,1, \mathrm{~F}=19,77, \mathrm{p}<0,001)$. No differences between the stages were observed for verbal tasks.
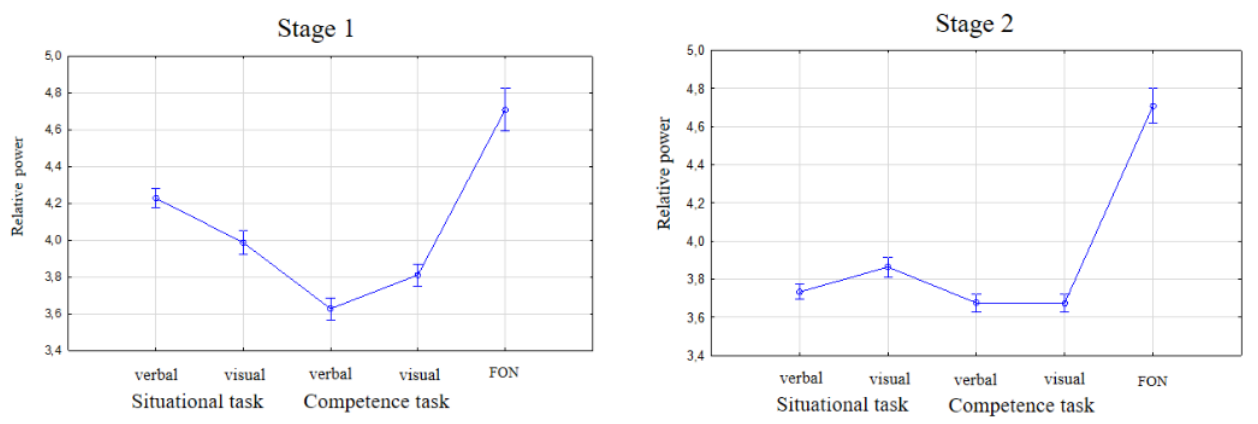

Fig. 5. Mean values of the EEG spectral power in the delta-diapason at the stages 1 and 2 of solving of the tasks of all types at the influence of the CONDITION factor. Note: the abscissa shows the type of the task; ordinate shows the EEG spectral power.

The dynamics of the theta-diapason at the stages 1 and 2 is shown on the Fig. 6. The single-factor analysis of variance of the stage 1 revealed significant influence of the CONDITION $(\mathrm{F}(4,19)=14,25, \mathrm{p}=0,0001)$ and LOCATION $(\mathrm{F}(20,19)=35,35, \mathrm{p}=0,0001)$ factors. At the stage 1 of the situational task solving, considerably higher values of the EEG spectral power in the theta-diapason were observed in case of verbal tasks in comparison with visual ones $(p=0,00001)$. No differences were observed in case of the competence tasks. The analysis of the effect of the task complexity showed that switching from situational verbal tasks to the competence verbal tasks is followed by the decrease in the EEG spectral power $(\mathrm{F}(1,19)=33,27, \mathrm{p}=0,0001)$ though no difference was found for visual tasks.

At the stage 2 the significant influence of the CONDITION $((\mathrm{F}(4,19)=15,28, \mathrm{p}=0,0001)$ and LOCATION $((\mathrm{F}(20,19)=26,19, \mathrm{p}=0,0001)$ factors was also observed. The comparative single-factor analysis of variance showed that solving of the situational verbal tasks was associated with significantly lower EEG spectral power in the theta-diapason in comparison with the stage $1(\mathrm{~F}(1,19)=43,28, \mathrm{p}=0,02)$, though no differences were observed for the stages 1 and 2 of other types of tasks. 

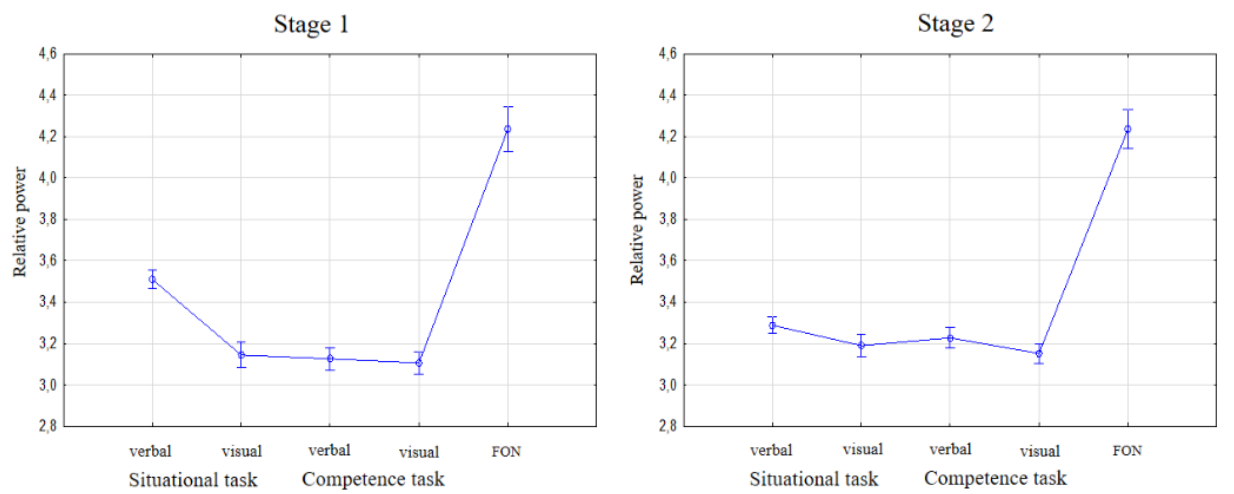

Fig. 6. Mean values of the EEG spectral power in the theta-diapason at the stages 1 and 2 of all types of tasks at the influence of the CONDITION factor. Note: the abscissa shows the type of the task; ordinate shows the EEG spectral power.

The dynamics of the alpha-diapason at the stages 1 and 2 is shown on the Fig. 7. The single-factor analysis of variance at the stage 1 revealed confident influence of the CONDITION $(\mathrm{F}(4,19)=22,45, \mathrm{p}=0,00041)$ and LOCATION $(\mathrm{F}(20,19)=57,2, \mathrm{p}=0,001)$ factors.

The stage 1 of the situational verbal task solving was characterized by higher EEG spectral power in the alpha-diapason in comparison with visual situational tasks $(F(1,19)=12,07, p=0,0005)$. No differences were found for the competence tasks. The assessment of the task complexity showed that switching from the verbal situational tasks to the competence verbal tasks was followed by the decrease in the EEG spectral power $(F(1,19)=43,27, p=0,0001)$, though no differences was found for the visual tasks.

Similar situation was observed for the stage 2. The significant effect of the LOCATION factor $(F(20,19)=64,07, p=0,0001)$ was also observed. Data of the single-factor analysis of variance, which was carried out in order to compare all types of tasks at the stage 2, did not reveal any differences. No confident difference was also observed in result of a comparative analysis of the stages 1 and 2 .
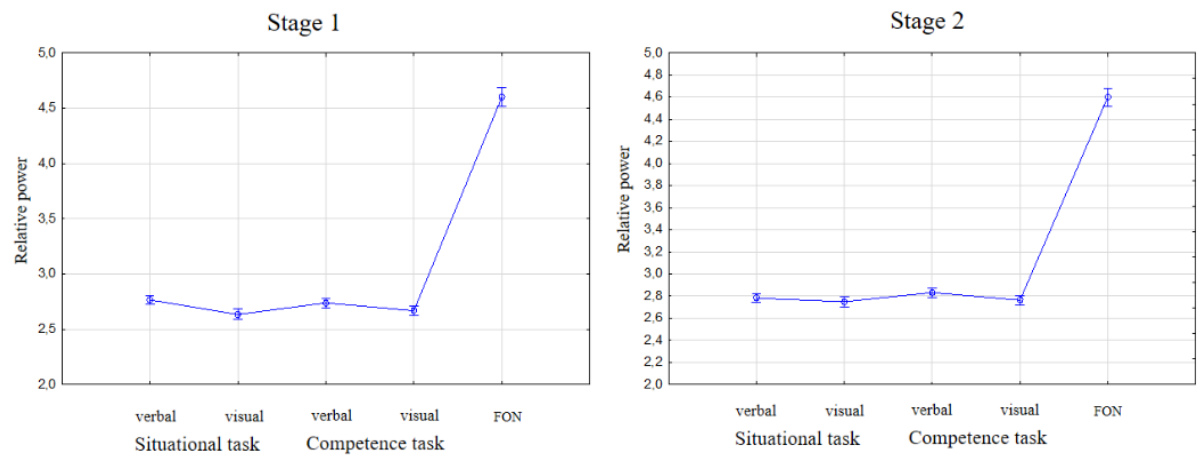

Fig. 7. Mean values of the EEG spectral power in the alpha-diapason at the stages 1 and 2 of all types of tasks at the influence of the CONDITION factor. Note: the abscissa shows the type of the task; ordinate shows the EEG spectral power.

\section{Discussion}

In the present study we modeled the situation of solving of real learning tasks under the conditions of psychophysiological examination. The addition of endogenous activity in the 
form of independent sort out of stages of learning task solving by a student allowed us to reveal their number and duration. It was previously shown that additional endogenous activity did not affect the time required for solving of simple tasks, though insignificantly increases the time of complex task solving $[4,5]$. Therefore, use of this activity as a reverse marker of cognitive task solving may be considered as acceptable.

A special feature of text learning tasks is low information value of the time required to solve the task. This is connected with big impact of a creative component into the solving of these tasks that requires simultaneous involvement of a number of cognitive processes. Therefore, we chose the first stage, which is connected with the task reading and the beginning of analysis, and combined second and third stages, which are connected with the main part of the task solving (the task rereading, consideration of the answer, text typing), in order to analyze the behavioral and electrophysiological parameters. This allowed us to suggest that different cognitive processes are involved at each stage.

The main factor, which affects the duration of individual stages of the task solving, is the complexity of the task. Solving of situational tasks was considerably less time-consuming in comparison with competence tasks. This confirms the suggestion of increasing complexity of the tasks in the "situational - competence" row regardless of their content. The main impact into the formation of this dynamics belongs to the first stage. Apparently, understanding of a competence task requires more cognitive resources. This leads to active involvement of the volitional attention system and working memory, which are connected with information saving and beginning of the task analysis. Solving of situational tasks is connected with the stage 1 . Hence, changes in its duration marks the involvement of the long-term memory, whereas the stage 2 marks the involvement of the working memory.

The number of terms used by a student in order to formulate the answer may be considered as the criterion of efficacy of activity at the stage 2 . However, use of this parameter is limited by the possibility to substitute the term with a synonym or use of general scientific vocabulary. The percentage of terms used to solve verbal tasks was considerably smaller in comparison with visual tasks. This is apparently caused by the very possibility itself to substitute the term with a synonym without decrease in the correctness of the answer. It is not possible in case of visual tasks, because of unambiguousness of anatomical terminology.

Use of this parameter allows us to assess the involvement of the long-term memory, because it is the very function the saving of the tasks during independent work is connected with. The correct selection of synonyms and general scientific vocabulary is also the marker of involvement of the working memory, in which the formation of the current variation of the answer takes place. This parameter is most applicable to creative tasks, because the content of standard tasks requires the formation of a concise answer.

The analysis of behavioral and electrophysiological parameters allowed us to conclude that solving of learning tasks of different complexity involves similar complex of cognitive processes: working memory, long-term memory and volitional attention. The main differences between the tasks were quantitative and connected with unequal impact of these processes into the task solving. This facilitated the activation of brain cortex and subcortical structures required for optimal solving of educational tasks. The differences revealed are apparently connected with a big number of other psychophysiological characteristics, including the level of current fuctional condition, situational motivation and individual level of learning competence.

The background values of the EEG spectral power decreased regardless of the task type so as to reach the level of general activity optimal for the task. Changes in the EEG spectral power in the theta- and alpha-frequencies, as well as their ratio, are considered as the correlate of learning and use of the standard educational rules [3,6]. Therefore, their similar dynamics confirms the involvement of a single set of cognitive processes into the task solving $[1,2,3]$. The increase in the delta-frequencies may be explained as a parameter of monitoring and 
inhibition of irrelevant information (cognitive control), as well as a parameter of the choice of an appropriate strategy to solve the task [7].

The main part of simple task solving was connected with the stage 1, at which confident changes in the EEG spectral power in the delta- and theta-diapasons were observed. This confirms activation of the long-term memory system at the stage 1, because of the necessity to recall the answer. The decrease in the EEG spectral power in the delta- and theta-diapasons at the stage 2 mirrored the leading role of the working memory system, in which the answer was fixed.

Solving of complex tasks proceeds at both stages and associated with activation of both types of memory, because of the necessity to synthesize information from different topics. One may admit the leading role of working memory, because of the absence of a ready standard answer in the long-term memory system. This is confirmed by predomination of the theta-frequencies in the EEG spectrum together with decreased expression of the alphadiapason. It is suggested that this ratio of frequency diapasons indicates involvement of corticohippocampus system and mirrors the coding of new information and formation of individual learning intensity [2, 8-12]. This was observed at the first stage of solving of both types of tasks regardless of the level of success. Non-specific reaction of alphadesynchronization created the required level of activation in order to involve the inner speech system $[6,13]$, phonological loop of the working memory system and its interaction with the long-term memory [14-18].

\section{Conclusion}

The analysis of behavioral and electrophysiological parameters allowed us to conclude that solving of learning tasks of different complexity involves similar complex of cognitive processes: working memory, long-term memory and volitional attention. The main differences between the tasks were quantitative and connected with unequal impact of these processes into the task solving. This facilitated the activation of brain cortex and subcortical structures required for optimal solving of educational tasks. The differences revealed are apparently connected with a big number of other psychophysiological characteristics, including the level of current functional condition, situational motivation and individual level of learning competence.

\section{References}

1. M.T. van Kesteren, M. Rijpkema, D.J. Ruiter, R.G. Morris, G. Fernández, J Cogn Neurosci 26(10), 2250-61 (2014) DOI: 10.1162 / jocn_a_00630

2. J.P. Kim, S.W. Seo, H.Y. Shin, B.S. Ye, J.J. Yang, et al, Neurology 85(9), 806-12 (2015) DOI: 10.1212 / WNL.0000000000001884

3. W. Skrandies, A. Klein, Brain research 1603, 133 -145 (2015) DOI: 10.1016 / j.brainres.2014.11.015

4. E.K. Aydarkin, A.S. Fomina, J Integr Neurosci. 12(1), $73-89$ (2013) DOI: 10.1142 / S0219635213500088

5. E.K. Aydarkin, O.L. Kundupyan, J.L. Kundupyan, J. Integr Neurosci. 12(1), 57-72 (2013) DOI: 10.1142 / S0219635213500076

6. B. De Smedt, R.H. Grabner, B. Studer, Exp Brain Res 195(4), 635-42 (2009) DOI: 10.1007 / s00221-009-1839-9

7. T. Hinault, P. Lemaire, Int J Psychophysiol. 106, 115-26 (2016) DOI: 10.1016 / j.ijpsycho.2016.05.006 
8. M. Pignatelli, A. Beyeler, X. Leinekugel, J Physiol Paris. 106, 81-92 (2012) DOI: 10.1016 / j.jphysparis.2011.09.007

9. C. Moisello, H.B. Meziane, S. Kelly, et al, PLoS One 8(6), 658-82 (2013) DOI: 10.1371 / journal.pone.0065882

10. J.S. Mayer, A. Roebroeck, K. Maurer, D.E. Linden, Human Brain Mapping 31(1), 126139 (2010) DOI: 10.1002 / hbm.20850

11. R. Panda, R.D. Bharath, L. George, Kanungo S, Reddy RP, Upadhyay N, Thamodharan A, Rajeshwaran J, Rao SL, Gupta AK.: Unraveling Brain Functional Connectivity of encoding and retrieval in the context of education. Et al, Brain Cogn. 86, 75-81 (2014) DOI: 10.1016 / j.bandc.2014.01.018

12. J. Kim, J. Chey, S.E. Kim, H. Kim, Neurosci Res. 94, 50-61 (2015) DOI: 10.1016 / j.neures.2014.12.009

13. S. Micheloyannis, V. Sakkalis, M. Vourkas, C. Stam, P. Simos, Neuroscience Letters 373, 212-217 (2005) DOI: 10.1016 / j.neulet.2004.10.005

14. O. Jensen, J.E. Lisman, Trends Neurosci. 2, 67-72 (2005) DOI: 10.1016 / j.tins.2004.12.001

15. M. Bastiaansen, P. Hagoort, Prog Brain Res. 159, 179-96 (2006) DOI: 10.1016 / S00796123 (06) 59012-0

16. A.K. Engel, Curr Opin Neurobiol. 20(2), 156-65 (2010) DOI: 10.1016 / j.conb.2010.02.015

17. A.E. Foley, M. Vasilyeva, E.V. Laski, Br J Dev Psychol. 35(2), 303-309 (2017) DOI: 10.1111 / bjdp. 12166

18. F. Vallée-Tourangeau, M. Sirota, G. Vallée-Tourangeau, Cogn Res Princ Implic. 1, 14 (2016) DOI: 10.1186 / s41235-016-0027-2 